\title{
Existence and regularity of the pressure for the stochastic Navier-Stokes equations
}

\author{
J.A. LANGA ${ }^{\dagger *}$ J. REAL ${ }^{\dagger}$ and J. Simon ${ }^{\ddagger}$
}

April 25, 2003

\begin{abstract}
We prove, on one hand, that for a convenient body force with values in the distribution space $\left(H^{-1}(D)\right)^{d}$, where $D$ is the geometric domain of the fluid, there exist a velocity $u$ and a pressure $p$ solution of the stochastic Navier-Stokes equation in dimension 2, 3 or 4 .

On the other hand, we prove that, for a body force with values in the dual space $V^{\prime}$ of the divergence free subspace $V$ of $\left(H_{0}^{1}(D)\right)^{d}$, in general it is not possible to solve the stochastic Navier-Stokes equations. More precisely, although such body forces have been considered, there is no topological space in which Navier-Stokes equations could be meaningful for them.
\end{abstract}

AMS Subject Classification (2000): 60H15, 60H30, 35R15, 35Q30

\section{Introduction}

Let $D$ be a connected and bounded open subset of $\mathbb{R}^{d}$, where $d=2,3$ or 4 , with a regular enough boundary $\partial D$. Let us fix a final time $T>0$, and consider the following system of stochastic Navier-Stokes equations with homogeneous Dirichlet boundary condition:

\footnotetext{
*Corresponding author

${ }^{\dagger}$ Department of Differential Equations and Numerical Analysis, University of Sevilla, Tarfia s/n, E-41012 Sevilla, Spain. e-mail: langa@numer.us.es; real@numer.us.es Fax: 95 4552898 Partially supported by MCYT (Feder), Proyecto BFM2002-03068

${ }^{\ddagger}$ Laboratoire de Mathématiques Appliquées, CNRS and Université Blaise Pascal, 63177 Aubière cedex, France. e-mail: Jacques.Simon@math.univ.bp-clermont.fr
} 


$$
\left\{\begin{array}{l}
\partial_{t} u-\nu \Delta u+(u \cdot \nabla) u+\nabla p=F(\cdot, u)+G(\cdot, u) \dot{W}_{t}, \text { in } D \times(0, T), \\
\nabla \cdot u=0, \text { in } D \times(0, T), \\
u=0, \text { on } \partial D \times(0, T),
\end{array}\right.
$$

where $u=\left(u_{1}, \ldots, u_{d}\right)$ and $p$ are unknown random fields on $D \times[0, T]$, representing respectively the velocity and the pressure of an incompressible fluid filling the domain $D$, in each point of $D \times[0, T]$ (in fact, $p$ is the sum of the pressure and of some potential $q$ corresponding to the part of forces of the form $\nabla q$ ). Here, the body force $F$ is a given measurable sublinear mapping from $[0, T] \times\left(L^{2}(D)\right)^{d}$ into $\left(H^{-1}(D)\right)^{d}, W$ is a cylindrical $K$-valued Wiener process on a complete probability space $(\Omega, \mathcal{F}, P)$, where $K$ is a fixed separable Hilbert space, $G$ is a given measurable sublinear mapping from $[0, T] \times\left(L^{2}(D)\right)^{d}$ into $\mathbb{L}^{2}\left(K ;\left(L^{2}(D)\right)^{d}\right)$ and $\nu>0$ is the kinematic viscosity of the fluid, which is constant.

Existence result. As we will see in Theorem 2.2, for such data, there exist a solution $(u, p)$ to (1). Similar results were obtained in [1], [3], [4], [5] and [6] among others. Our contribution here is that we obtain the pressure $p$ without any regularity assumption on $u$, contrarily to [5] or [6] in which its values are assumed to be in $\left(H^{2}(D)\right)^{d}$, and that we get equation $(1)$ in the distribution sense, contrarily to the case where the body force is valued in $V^{\prime}$ in which case no satisfactory sense can be given, see Theorem 6.1.

As in most quoted papers, we obtain the solution in two steps. First, we consider a velocity $u$ satisfying $P$-a.s. the following so called "variational $\mathrm{N}-\mathrm{S}$ equation" (in which the pressure is eliminated): for all $t \in[0, T]$ and for all $v \in(\mathcal{D}(D))^{d}$ such that $\nabla \cdot v=0$,

$$
\begin{array}{r}
\int_{D} u(t) \cdot v d x=\int_{D} u(0) \cdot v d x-\nu \int_{0}^{t} \int_{D} \nabla u(s) \cdot \nabla v d x d s \\
-\int_{0}^{t} \int_{D}(u(s) \cdot \nabla u(s)) \cdot v d x d s+\int_{0}^{t}\langle F(s, u(s)), v\rangle_{H^{-1}(D) \times H_{0}^{1}(D)} d s \\
+\int_{D} \int_{0}^{t} G(s, u(s)) d W_{s} \cdot v d x .
\end{array}
$$

The existence of such a $u$ is proved in [6]; the first result in this direction was given in [2] in the case of $K=\mathbb{R}$ and $G$ constant; it was extended to a multiplicative noise and to an infinite-dimensional $K$ in [1], [3], [4], [5] and [6] among others.

In the second step, we associate a pressure $p$ to such a $u$ by using a generalization of de Rham theorem to processes, see Theorem 4.1. 
Non-existence result. Various authors considered a body force $F$ with values in the dual $V^{\prime}$ of the space $V=\left\{v \in\left(H_{0}^{1}(D)\right)^{d}: \nabla \cdot v=0\right\}$ instead of $\left(H^{-1}(D)\right)^{d}$ as above. Then they solve, again for all $v \in(\mathcal{D}(D))^{d}$ such that $\nabla \cdot v=0$ (or equivalently for all $v \in V$ ),

$$
\begin{array}{r}
\int_{D} u(t) \cdot v d x=\int_{D} u(0) \cdot v d x-\nu \int_{0}^{t} \int_{D} \nabla u(s) \cdot \nabla v d x d s \\
-\int_{0}^{t} \int_{D}(u(s) \cdot \nabla u(s)) \cdot v d x d s+\int_{0}^{t}\langle F(s, u(s)), v\rangle_{V^{\prime} \times V} d s \\
+\int_{D} \int_{0}^{t} G(s, u(s)) d W_{s} \cdot v d x
\end{array}
$$

that is (2) in which the duality $H^{-1} \times H_{0}^{1}$ is replaced by the duality $V^{\prime} \times V$.

Unfortunately, as we will see in Theorem 6.1 , it cannot exist any $p$ corresponding to such $u$, or more exactly to such $F$, such that the first equation in (1) be satisfied. Indeed, $F$ being valued in $V^{\prime}$ while other terms are valued in $\left(H^{-1}(\Omega)\right)^{d}$, it would be necessary to imbed these two spaces in a same Hausdorff space, which is impossible.

Similar existence and non-existence results for the deterministic NavierStokes equations may be found in [12].

\section{Existence of a solution $(u, p)$ of the stochastic Navier-Stokes equations.}

In all the sequel, let

$D$ be a bounded, connected and Lipschitz open subset of $\mathbb{R}^{d}$,

$$
d \in\{2,3,4\},
$$

$K$ be a separable Hilbert space.

Let $F$ and $G$ be two mappings such that

$$
\left\{\begin{array}{l}
F \text { is measurable from }[0, T] \times\left(L^{2}(D)\right)^{d} \text { into }\left(H^{-1}(D)\right)^{d}, \\
G \text { is measurable from }[0, T] \times\left(L^{2}(D)\right)^{d} \text { into } \mathbb{L}^{2}\left(K ;\left(L^{2}(D)\right)^{d}\right),
\end{array}\right.
$$

and, for all $t \in[0, T], w \in(\mathcal{D}(D))^{d}$ and $e \in K$,

$$
\left\{\begin{array}{l}
v \mapsto\langle F(t, v), w\rangle_{\left(\mathcal{D}^{\prime}(D)\right)^{d} \times(\mathcal{D}(D))^{d}} \text { and } v \mapsto \int_{D} G(t, v) e \cdot w d x \\
\text { are continuous from }\left(L^{2}(D)\right)^{d} \text { into } \mathbb{R}
\end{array}\right.
$$


and such that there exists a positive number $c_{1}$ such that, for all $t \in[0, T]$ and $v \in\left(L^{2}(D)\right)^{d}$,

$$
\left\{\begin{array}{l}
\|F(t, v)\|_{\left(H^{-1}(D)\right)^{d}} \leq c_{1}\left(1+\|v\|_{\left(L^{2}(D)\right)^{d}}\right), \\
\|G(t, v)\|_{\mathbf{L}^{2}\left(K ;\left(L^{2}(D)\right)^{d}\right)} \leq c_{1}\left(1+\|v\|_{\left(L^{2}(D)\right)^{d}}\right) .
\end{array}\right.
$$

Let us denote by $H$ the closure of the set

$$
\mathcal{V}=\left\{v \in(\mathcal{D}(D))^{d}: \nabla \cdot v=0 \text { in } D\right\}
$$

in $\left(L^{2}(D)\right)^{d}$, and by $V$ the closure of $\mathcal{V}$ in $\left(H^{1}(D)\right)^{d}$. Then, $H$ is a Hilbert space equipped with the inner product of $\left(L^{2}(D)\right)^{d}$, and $V$ is a Hilbert space equipped with the inner product of $\left(H^{1}(D)\right)^{d}$. Finally, let

$$
\left\{\begin{array}{c}
\mu \text { be a probability measure on } H \text { such that, for all } r \in[1, \infty), \\
\int_{H}\|v\|_{H}^{r} d \mu(v)<\infty .
\end{array}\right.
$$

Definition 2.1 A martingale solution to (1) starting from $\mu$ is a set $\left\{(\Omega, \mathcal{F}, P),\left\{\mathcal{F}_{t}\right\}_{t \in[0, T]}, W, u, p\right\}$, such that:

$(\Omega, \mathcal{F}, P)$ is a probability space,

$\left\{\mathcal{F}_{t}\right\}_{t \in[0, T]}$ is a normal filtration on $(\Omega, \mathcal{F}, P)$,

$W$ is a cylindrical $K$-valued $\mathcal{F}_{t}$-Wiener process,

$$
\begin{gathered}
u \in M_{\mathcal{F}_{t}}^{2}(0, T ; V) \cap L^{r}\left(\Omega, \mathcal{F}, P ; L^{\infty}(0, T ; H)\right), \quad \forall r \in[1, \infty), \\
p \in L^{1}\left(\Omega, \mathcal{F}_{t}, P ; W^{-1, \infty}\left(0, t ; L^{2}(D)\right)\right), \quad \forall t \in(0, T], \\
P\{u(0) \in B\}=\mu(B), \quad \forall B \in \mathcal{B}(H),
\end{gathered}
$$

and such that, $P$-a.s.:

$$
\begin{gathered}
\partial_{t} u-\nu \Delta u+(u \cdot \nabla) u+\nabla p=F(\cdot, u)+G(\cdot, u) \dot{W}_{t}, \quad \text { in }\left(\mathcal{D}^{\prime}\left(D_{T}\right)\right)^{d}, \\
\nabla \cdot u=0, \quad \text { in } \mathcal{D}^{\prime}\left(D_{T}\right), \\
\int_{D} p d x=0, \quad \text { in } \mathcal{D}^{\prime}(0, T), \\
u \in \mathcal{C}\left([0, T] ; H \text {-weak } \cap\left(H^{-1}(D)\right)^{d}\right),
\end{gathered}
$$

where $D_{T}=(0, T) \times D$.

We are now in position to state the existence result of a solution $(u, p)$ to the stochastic Navier-Stokes equations (1): 
Theorem 2.2 Assuming (4) to (10), there exists a martingale solution to (1) starting from $\mu$.

Remark 2.3 In view of Theorem 4.1, to any such velocity $u$, it corresponds a unique pressure $p$.

Many papers consider martingale solutions of the variational equation (2) in which $p$ is eliminated, see for example [6]. Again in view of Theorem 4.1, assuming (4) to (10), it is equivalent to the existence of a martingale solution to (1).

\section{Some definitions.}

In this section, we recall the definitions of some above used properties.

\subsection{Lipschitz domain, used in (4)}

A non empty open subset $D$ of $\mathbb{R}^{d}$ is said Lipschitz if it is locally the epigraph of a Lipschitz function. Or, more precisely, if there exist two positive numbers $a$ and $\kappa$ such that, for each point $\xi \in \partial D$, there exists a system of cartesian coordinates $\left(x_{1}, \ldots, x_{d}\right)$ with origin at $\xi$ and a real function $\psi$ defined on $O^{\prime}=\left\{x^{\prime} \in \mathbb{R}^{d-1}:\left|x^{\prime}\right|<a\right\}$, where $x^{\prime}=\left(x_{1}, \ldots, x_{d-1}\right)$, such that, for all $x^{\prime}$ and $y^{\prime}$ in $O^{\prime}$,

$$
\left|\psi\left(x^{\prime}\right)-\psi\left(y^{\prime}\right)\right| \leq \kappa\left|x^{\prime}-y^{\prime}\right|,
$$

and, for all $x \in O=\left\{x=\left(x^{\prime}, x_{d}\right) \in \mathbb{R}^{d}:\left|x^{\prime}\right|<a,\left|x_{d}\right|<a\right\}$,

$$
\begin{aligned}
x \in D & \Longleftrightarrow x_{d}<\psi\left(x^{\prime}\right), \\
x \in \partial D & \Longleftrightarrow x_{d}=\psi\left(x^{\prime}\right) .
\end{aligned}
$$

\subsection{Normal filtration and Wiener process, used in Def. (2.1)}

A normal filtration on a propability space $(\Omega, \mathcal{F}, P)$ is an increasing and right continuous family $\left\{\mathcal{F}_{t}\right\}_{t \in[0, T]}$ of sub $\sigma$-algebras of $\mathcal{F}$, such that $\mathcal{F}_{0}$ contains all the $P$ null sets of $\mathcal{F}$.

Given a separable Hilbert space $K$, a cylindrical $K$-valued $\mathcal{F}_{t}$-Wiener process is any "process" $W$ formally defined as

$$
W_{t}=\sum_{i=1}^{\infty} \beta_{t}^{i} e_{i},
$$


where $\left(\beta_{t}^{i}: t \geq 0, i=1,2, \ldots\right)$ are mutually independent standard real $\mathcal{F}_{t}$-Wiener processes defined on $(\Omega, \mathcal{F}, P)$, and $\left\{e_{i}: i=1,2, \ldots\right\}$ is an orthonormal basis of $K$. It is well known that the series defining $W$ does not converge in $K$, but rather in any Hilbert space $\tilde{K}$ such that $K \subset \tilde{K}$ and the injection of $K$ in $\tilde{K}$ is Hilbert-Schmidt (see e.g. [7]).

\subsection{Mesurability and $\mathbb{L}^{2}$ space, used in (7)}

Let $X$ and $Y$ be Banach spaces and let $\mathcal{B}(X)$ and $\mathcal{B}(Y)$ be the $\sigma$-algebras of Borel subsets respectively of $X$ and $Y$. A map from $h: X \rightarrow Y$ is said measurable if $h^{-1}(B) \in \mathcal{B}(X)$ for all $B \in \mathcal{B}(Y)$.

Given now another (than $K$ ) separable Hilbert space $U$, with inner product $(\cdot, \cdot)_{U}$, we denote by $\mathbb{L}^{2}(K ; U)$ the space of Hilbert-Schmidt operators from $K$ into $U$ provided with the Hilbert norm associated to the scalar product defined, for all $A$ and $B$ in $\mathbb{L}^{2}(K ; U)$ by

$$
(A, B)_{\mathbf{L}^{2}(K ; U)}=\sum_{k=1}^{\infty}\left(A e_{k}, B e_{k}\right)_{U}
$$

where $\left\{e_{k}\right\}_{k=1}^{\infty}$ is a Hilbert basis of $K$.

\section{$3.4 \quad L^{r}$ and $M_{\mathcal{F}_{t}}^{2}$ spaces, used in (11)}

Let again $X$ and $Y$ be Banach spaces. Given a $\sigma$-algebra $\mathcal{G} \subset \mathcal{F}$, we denote by $\mathcal{L}^{0}(\Omega, \mathcal{G}, P ; Y)$ the vector space of all the mappings $h: \Omega \rightarrow Y$ that are $\mathcal{G}$-measurables, i.e., such that $h^{-1}(B) \in \mathcal{G}$ for all $B \in \mathcal{B}(Y)$. We denote by $L^{0}(\Omega, \mathcal{G}, P ; Y)$ the vector space of equivalence classes of mappings in $\mathcal{L}^{0}(\Omega, \mathcal{G}, P ; Y)$, differing only on a $P$-null set. For a given $r \in[1, \infty)$, we denote

$$
L^{r}(\Omega, \mathcal{G}, P ; Y)=\left\{h \in L^{0}(\Omega, \mathcal{G}, P ; Y): E\left(\|h\|_{Y}^{r}\right)<\infty\right\}
$$

where $E$ stands for the expectation. Analogously, we denote

$$
L^{\infty}(\Omega, \mathcal{G}, P ; Y)=\left\{h \in L^{0}(\Omega, \mathcal{G}, P ; Y):\|h\|_{Y} \in L^{\infty}(\Omega, \mathcal{G}, P)\right\} .
$$

In particular, as usual, given an open subset $\mathcal{O}$ of $\mathbb{R}^{n}$, we denote $L^{r}(\mathcal{O} ; Y)=$ $L^{r}(\mathcal{O}, \mathcal{B}(\mathcal{O}), d x ; Y)$, where $d x$ is the Lebesgue measure, $L^{r}(\mathcal{O})=L^{r}(\mathcal{O} ; \mathbb{R})$ and $L^{r}(0, T ; Y)=L^{r}((0, T) ; Y)$.

Now, let us recall that an $\mathcal{F}_{t}$-progressively measurable stochastic process with values in $X$ is any stochastic process $z: \Omega \times[0, T] \rightarrow X$ such 
that, for all $t \in[0, T]$, the restriction of $z$ to $\Omega \times[0, t]$ is $\mathcal{F}_{t} \times \mathcal{B}([0, t])$ measurable. More generally, given $z \in L^{0}\left(\Omega, \mathcal{F}, P ; L^{1}(0, T ; X)\right)$, we say that $z$ is $\mathcal{F}_{t}$-progressively measurable if there exists an $\mathcal{F}_{t}$-progressively measurable stochastic process $\hat{z}$ with values in $X$ such that $\hat{z}=z, d P \times d t$-a.e. We denote

$$
M_{\mathcal{F}_{t}}^{2}(0, T ; X)=\left\{z \in L^{2}(\Omega \times(0, T), d P \times d t ; X): z \text { is } \mathcal{F}_{t} \text {-progr. meas. }\right\} .
$$

If $X$ is a Hilbert space, then the space $M_{\mathcal{F}_{t}}^{2}(0, T ; X)$ is a Hilbert subspace of $L^{2}(\Omega \times(0, T), d P \times d t ; X)$.

\subsection{Vector-valued distributions, used in (12)}

We now define distribution spaces because $W^{-1, \infty}\left(0, T ; L^{2}(D)\right)$, used in (12), will next be defined as a subspace of such a space.

Let again $\mathcal{O}$ be an open subset of $\mathbb{R}^{n}$, and let $\mathcal{Y}$ be a complete lcstvs, that is a locally convex separated topological vector space (the case where it is not a Banach space is used in (23)). The space of $\mathcal{Y}$-valued distributions on $\mathcal{O}$ is defined by

$$
\mathcal{D}^{\prime}(\mathcal{O} ; \mathcal{Y})=\mathcal{L}_{c}(\mathcal{D}(\mathcal{O}) ; \mathcal{Y})
$$

where $\mathcal{L}_{c}$ stands for linear continuous (here, it is equivalent to sequentially continuous) and $\mathcal{D}(\mathcal{O})$ is the space of indefinitely differentiable functions with a compact support included in $\mathcal{O}$. As usually, we denote $\mathcal{D}^{\prime}(\mathcal{O})=$ $\mathcal{D}^{\prime}(\mathcal{O} ; \mathbb{R}), \mathcal{D}^{\prime}(0, T ; \mathcal{Y})=\mathcal{D}^{\prime}((0, T) ; \mathcal{Y})$ and $\mathcal{D}(0, T)=\mathcal{D}((0, T))$. Given $f \in$ $\mathcal{D}^{\prime}(\mathcal{O} ; \mathcal{Y})$ and $\varphi \in \mathcal{D}(\mathcal{O})$, we frequently denote $\langle f, \varphi\rangle_{\mathcal{D}^{\prime}(\mathcal{O} ; \mathcal{Y}) \times \mathcal{D}(\mathcal{O})}=f(\varphi)$.

Given $f \in \mathcal{C}(\mathcal{O} ; \mathcal{Y})$ we identify it to the distribution $\dot{f}$ defined by

$$
\langle\dot{f}, \varphi\rangle_{\mathcal{D}^{\prime}(\mathcal{O} ; \mathcal{Y}) \times \mathcal{D}(\mathcal{O})}=\int_{\mathcal{O}} \varphi f d x, \quad \forall \varphi \in \mathcal{D}(\mathcal{O}) .
$$

This provides a topological imbedding $\mathcal{C}(\mathcal{O} ; Y) \subset \mathcal{D}^{\prime}(\mathcal{O} ; Y)$. The completeness (it could be relaxed in sequential completeness) of $\mathcal{Y}$ is assumed in order to get this imbedding which is essential (else, the space $\mathcal{L}_{c}(\mathcal{D}(\mathcal{O}) ; \mathcal{Y})$ is still defined but it no longer "contains" continuous functions, and therefore it must not be denoted $\mathcal{D}^{\prime}$ and its elements must not be named "distributions").

\subsection{Sobolev spaces, used in (7) and (12)}

Let again $\mathcal{O}$ be an open subset of $\mathbb{R}^{n}, Y$ be a Banach space, and $r \in[1, \infty]$. Given $f \in L^{r}(\mathcal{O} ; Y)$ we identify it to the distribution $\dot{f}$ again defined by (19). 
This provides a topological imbedding $L^{r}(\mathcal{O} ; Y) \subset \mathcal{D}^{\prime}(\mathcal{O} ; Y)$ and allows to define the derivatives of $f$ to be $\partial_{i} \dot{f}$, where $\partial_{i}$ stands for $\partial / \partial x_{i}$.

Now, we can define

$$
\begin{gathered}
W^{1, r}(\mathcal{O} ; Y)=\left\{f \in L^{r}(\mathcal{O} ; Y): \partial_{i} f \in L^{r}(\mathcal{O} ; Y), 1 \leq i \leq n\right\}, \\
W^{-1, r}(\mathcal{O} ; Y)=\left\{f \in \mathcal{D}^{\prime}(\mathcal{O} ; Y): f=f_{0}+\sum_{i=1}^{n} \partial_{i} f_{i}, f_{i} \in L^{r}(\mathcal{O} ; Y), 0 \leq i \leq n\right\} .
\end{gathered}
$$

These spaces are respectively endowed with the norms

$$
\begin{gathered}
\|f\|_{W^{1, r}(\mathcal{O} ; Y)}=\left(\left(\|f\|_{L^{r}(\mathcal{O} ; Y)}\right)^{r}+\sum_{i=1}^{n}\left(\left\|\partial_{i} f\right\|_{L^{r}(\mathcal{O} ; Y)}\right)^{r}\right)^{1 / r}, \\
\|f\|_{W^{-1, r}(\mathcal{O} ; Y)}=\inf \left(\sum_{i=0}^{n}\left(\left\|f_{i}\right\|_{L^{r}(\mathcal{O} ; Y)}\right)^{r}\right)^{1 / r},
\end{gathered}
$$

where the infimum is taken for all the decompositions of $f$. As usual, $H^{1}$ stands for $W^{1,2}, H^{-1}$ for $W^{-1,2}$, and $H^{s}(\mathcal{O})$ for $H^{s}(\mathcal{O} ; \mathbb{R})$.

The spaces $W^{1, r}(\mathcal{O} ; Y)$ and $W^{-1, r}(\mathcal{O} ; Y)$ are Banach spaces; they are separables if $Y$ is separable and $r<\infty$. The spaces $H^{1}(\mathcal{O} ; Y)$ and $H^{-1}(\mathcal{O} ; Y)$ are Hilbert spaces if $Y$ is a Hilbert space.

Let $H_{0}^{1}(\mathcal{O})$ be the closure of $\mathcal{D}(\mathcal{O})$ in $H^{1}(\mathcal{O})$. Then, their dual spaces satisfy $\left(H_{0}^{1}(\mathcal{O})\right)^{\prime} \subset(\mathcal{D}(\mathcal{O}))^{\prime}$ with continuous imbedding. In fact, see for example [8] Theorem 5.1 p. 19,

$$
\left(H_{0}^{1}(\mathcal{O})\right)^{\prime}=H^{-1}(\mathcal{O}) .
$$

Moreover, the duality pairing satisfies, for all $f \in H^{-1}(D)$ and $\varphi \in H_{0}^{1}(\mathcal{O})$,

$$
\langle f, \varphi\rangle_{H^{-1}(D) \times H_{0}^{1}(D)}=\langle f, \varphi\rangle_{\mathcal{D}^{\prime}(D) \times \mathcal{D}(D)} .
$$

This duality property is frequently used to define $H^{-1}$. More generally, if $1<r<\infty$ and $Y$ is reflexive, then $W^{-1, r}(\mathcal{O} ; Y)=\left(W_{0}^{1, r^{\prime}}\left(\mathcal{O} ; Y^{\prime}\right)\right)$, where $Y^{\prime}$ stands the dual space of $Y$ and $1 / r^{\prime}+1 / r=1$; it is not convenient to use this equality as a general definition for $W^{-1, r}$ because it does not provide the right space if $r=1$ or $Y$ is not reflexive.

\subsection{Linear image of a distribution, used in (16)}

Let $\mathcal{Y}$ and $\mathcal{X}$ be two complete lcstvs and let $A \in \mathcal{L}_{c}(\mathcal{Y} ; \mathcal{X})$. Given $f \in$ $\mathcal{D}^{\prime}(\mathcal{O} ; \mathcal{Y})$, its image $A f \in \mathcal{D}^{\prime}(\mathcal{O} ; \mathcal{X})$ is defined by

$$
(A f)(\varphi)=A(f(\varphi)), \quad \forall \varphi \in \mathcal{D}(\mathcal{O}) .
$$


In the case of Banach spaces, $A$ maps continuously $L^{r}(\mathcal{O} ; \mathcal{Y}), W^{1, r}(\mathcal{O} ; \mathcal{Y})$ and $W^{-1, r}(\mathcal{O} ; \mathcal{Y})$ respectively into $L^{r}(\mathcal{O} ; \mathcal{X}), W^{1, r}(\mathcal{O} ; \mathcal{X})$ and $W^{-1, r}(\mathcal{O} ; \mathcal{X})$.

Now let us examine (16). It reads $\int_{D} p(\omega) d x=0$ for $P$-almost all $\omega \in \Omega$. It is meaningful since, $p(\omega)$ lying in $W^{-1, \infty}\left(0, T ; L^{2}(D)\right)$ by $(12)$, its image by the map $\int_{D} \in \mathcal{L}_{c}\left(L^{2}(D) ; \mathbb{R}\right)$ is defined by $(22)$ and satisfies

$$
\int_{D} p(\omega) d x \in W^{-1, \infty}(0, T ; \mathbb{R}) .
$$

\subsection{Separation of variables, used in (11) and (12)}

The separation of variable for functions, which maps $\mathcal{C}((0, T) \times D ; \mathcal{Y})$ onto $\mathcal{C}(0, T ; \mathcal{C}(D ; \mathcal{Y}))$, extends by continuity in a one-to-one bicontinuous map from $\mathcal{D}^{\prime}((0, T) \times D ; \mathcal{Y})$ onto $\mathcal{D}^{\prime}\left(0, T ; \mathcal{D}^{\prime}(D ; \mathcal{Y})\right)$ (the surjectivity, which is the hard point, is related to Schwartz's kernel theorem, see [9] for real values). Using this map to identify the spaces, we get the topological equality

$$
\mathcal{D}^{\prime}((0, T) \times D ; \mathcal{Y})=\mathcal{D}^{\prime}\left(0, T ; \mathcal{D}^{\prime}(D ; \mathcal{Y})\right)
$$

This identity allows us to consider $u(\omega)$ and $p(\omega)$ either as distributions on $(0, T) \times D$, as in (14) and (15), or as distributions on $(0, T)$ with values in a space of distributions on $D$, as in (11) and (12).

\subsection{Nonlinear term $(u \cdot \nabla) u$, used in (14)}

We denote $\nabla=\left(\partial_{1}, \ldots, \partial_{d}\right)$ the spatial gradient. Then, given $u=\left(u_{1}, \ldots, u_{d}\right)$, its divergence, used in (15), is $\nabla \cdot u=\partial_{1} u_{1}+\cdots+\partial_{d} u_{d}$.

Similarly, $u \cdot \nabla=u_{1} \partial_{1}+\cdots+u_{d} \partial_{d}$. Then, given $u$ and $v$ in $\left(H^{1}(D)\right)^{d}$, we define $(u \cdot \nabla) v$ to be the vector function which components are the $\sum_{j=1}^{d} u_{j} \partial_{j} v_{i}$, for $i=1, \ldots, d$. Since $d \leq 4$ and $D$ is Lipschitz, by Sobolev theorem, $H^{1}(D) \subset L^{4}(D)$ with continuous injection. Then, by Hölder inequality, the map $(u, v) \mapsto(u \cdot \nabla) v$ is bilinear continuous from $\left(\left(H^{1}(D)\right)^{d}\right)^{2}$ into $\left(L^{4 / 3}(D)\right)^{d}$, and therefore from $\left(L^{2}(0, T ; V)\right)^{2}$ into $L^{1}\left(0, T ;\left(L^{4 / 3}(D)\right)^{d}\right)$.

\subsection{Time-derivative $G \dot{W}_{t}$, used in (14)}

Let $U$ be a separable Hilbert space. Given $G \in M_{\mathcal{F}_{t}}^{2}\left(0, T ; \mathbb{L}^{2}(K ; U)\right)$, its Itô's stochastic integral with respect to the cylindrical Wiener process $W$, denoted $\left\{\int_{0}^{t} G_{s} d W_{s}: 0 \leq t \leq T\right\}$ is defined to be the unique continuous $U$-valued $\mathcal{F}_{t}$-martingale such that, for all $g \in U$ and $t \in[0, T]$,

$$
\left(\int_{0}^{t} G_{s} d W_{s}, g\right)_{U}=\sum_{i=1}^{\infty} \int_{0}^{t}\left(G_{s} e_{k}, g\right)_{U} d \beta_{s}^{i}
$$


where each stochastic integral in the series is understood as an Itô's stochastic integral with respect to the corresponding real valued Wiener process. The series in $(24)$ converges in $L^{2}\left(\Omega, \mathcal{F}_{t}, P ; \mathcal{C}([0, t])\right)$, for each $t \in(0, T]$, see [7] for details. Since $\int_{0}^{\cdot} G_{s} d W_{s} \in L^{2}\left(\Omega, \mathcal{F}_{t}, P ; \mathcal{C}([0, t] ; U)\right)$, its timederivative, that formally we will denote $G \dot{W}_{t}$, satisfies

$$
G \dot{W}_{t} \in L^{2}\left(\Omega, \mathcal{F}_{t}, P ; W^{-1, \infty}(0, t ; U)\right), \quad \forall t \in(0, T],
$$

because $\partial_{t}$ is linear continuous from $\mathcal{C}$, and therefore from $L^{\infty}$, into $W^{-1, \infty}$.

This applies here with $U=\left(L^{2}(D)\right)^{d}$, because, thanks to (7), (8), (9) and $(11), G(\cdot, u) \in M_{\mathcal{F}_{t}}^{2}\left(0, T ; \mathbb{L}^{2}\left(K ;\left(L^{2}(D)\right)^{d}\right)\right)$. Then,

$$
G(\cdot, u) \dot{W}_{t} \in L^{2}\left(\Omega, \mathcal{F}_{t}, P ; W^{-1, \infty}\left(0, t ;\left(L^{2}(D)\right)^{d}\right)\right), \quad \forall t \in(0, T] .
$$

\section{A generalization of de Rham theorem to pro- cesses.}

We will associate a pressure $p$ to a solution $u$ of the variational Navier-Stokes equation by the following result.

Theorem 4.1 Let $D$ satisfy $(4),(\Omega, \mathcal{G}, P)$ be a complete probability space and, given $r_{0} \in[1, \infty], r_{1} \in[1, \infty]$ and $s_{1} \in \mathbb{Z}$, let

$$
h \in L^{r_{0}}\left(\Omega, \mathcal{G}, P ; W^{s_{1}, r_{1}}\left(0, T ;\left(H^{-1}(D)\right)^{d}\right)\right)
$$

be such that, for all $v \in(\mathcal{D}(D))^{d}$ such that $\nabla \cdot v=0$, P-a.s.,

$$
\langle h, v\rangle_{\left(\mathcal{D}^{\prime}(D)\right)^{d} \times(\mathcal{D}(D))^{d}}=0, \quad \text { in } \mathcal{D}^{\prime}(0, T) .
$$

Then, there exists a unique

$$
p \in L^{r_{0}}\left(\Omega, \mathcal{G}, P ; W^{s_{1}, r_{1}}\left(0, T ; L^{2}(D)\right)\right)
$$

such that, P-a.s.,

$$
\begin{gathered}
\nabla p=h, \quad \text { in }\left(\mathcal{D}^{\prime}((0, T) \times D)\right)^{d}, \\
\int_{D} p d x=0, \quad \text { in } \mathcal{D}^{\prime}(0, T) .
\end{gathered}
$$

Moreover, there exists a positive number $c_{2}(D)$, independent of $h$, such that, P-a.s.,

$$
\|p\|_{W^{s_{1}, r_{1}\left(0, T ; L^{2}(D)\right)} \leq} \leq c_{2}(D)\|h\|_{W^{s_{1}, r_{1}\left(0, T ;\left(H^{-1}(D)\right)^{d}\right)}} .
$$


Proof. Let

$$
E=\left\{w \in\left(H^{-1}(D)\right)^{d}:\langle w, v\rangle_{\left(\mathcal{D}^{\prime}(D)\right)^{d} \times(\mathcal{D}(D))^{d}}=0, \forall v \in \mathcal{V}\right\}
$$

be equipped with the norm of $\left(H^{-1}(D)\right)^{d}$. Given $w \in E$, there exists a unique $q \in L^{2}(D)$ such that $\nabla q=w$ and $\int_{D} q d x=0$ and there exists a positive number $c_{2}(D)$, independent of $w$, such that

$$
\|q\|_{L^{2}(D)} \leq c_{2}(D)\|w\|_{\left(H^{-1}(D)\right)^{d}} .
$$

Indeed, by de Rham theorem, see for example [10], there exists $q_{1} \in L^{2}(D)$ such that $\nabla q_{1}=w$. Moreover, see for example Theorem 14 in [11], thanks to hypothesis (4) on $D, \nabla q_{1} \in\left(H^{-1}(D)\right)^{d}$ implies that $q_{1} \in L^{2}(D)$ and $\left\|q_{1}-\frac{1}{|D|} \int_{D} q_{1}\right\|_{L^{2}(D)} \leq c_{2}(D)\|w\|_{\left(H^{-1}(D)\right)^{d}}$. Then, $q=q_{1}-\frac{1}{|D|} \int_{D} q_{1}$ satisfies (32). Its uniqueness is obvious since $D$ is connected.

Then, we define a continuous linear map $A$ from $E$ into $L^{2}(D)$ by $A w=$ $q$. It satisfies, for all $w \in E$,

$$
\nabla A w=w, \quad \int_{D} A w d x=0 .
$$

Now, let us give two properties that hold for $P$-almost all $\omega \in \Omega$. First, (27) gives, by definition (22), for all $\varphi \in \mathcal{D}(0, T)$,

$$
\langle(h(\omega))(\varphi), v\rangle_{\left(\mathcal{D}^{\prime}(D)\right)^{d} \times(\mathcal{D}(D))^{d}}=0,
$$

that is, $(h(\omega))(\varphi) \in E$. Second, by $(26), h(\omega) \in W^{s_{1}, r_{1}}\left(0, T ;\left(H^{-1}(D)\right)^{d}\right)$. Since $E$ is closed in $\left(H^{-1}(D)\right)^{d}$, these two properties give, by the first property of the following Lemma 4.2, for $P$-almost all $\omega \in \Omega$,

$$
h(\omega) \in W^{s_{1}, r_{1}}(0, T ; E) .
$$

Since $W^{s_{1}, r_{1}}(0, T ; E)$ is closed in $W^{s_{1}, r_{1}}\left(0, T ;\left(H^{-1}(D)\right)^{d}\right),(26)$ and $(34)$ give, now by the second property of Lemma 4.2 ,

$$
h \in L^{r_{0}}\left(\Omega, \mathcal{G}, P ; W^{s_{1}, r_{1}}(0, T ; E)\right) .
$$

Then, thanks to (32) and (33), its image $p=A h$ satisfies (28) to (31), see Section 3.7.

To complete the proof, it remains to check the following properties. 
Lemma 4.2 Given a closed subspace $F$ of a Banach space $Y, r \in[1, \infty]$ and $s \in \mathbb{Z}$,

$$
\begin{gathered}
L^{r}(\Omega, \mathcal{G}, P ; F)=\left\{h \in L^{r}(\Omega, \mathcal{G}, P ; Y): h(\omega) \in F, \quad \text { P-a.s. } \omega \in \Omega\right\}, \\
W^{s, r}(0, T ; F)=\left\{h \in W^{s, r}(0, T ; Y): h(\varphi) \in F, \quad \text { for all } \varphi \in \mathcal{D}(0, T)\right\} .
\end{gathered}
$$

Proof. In view of definition (18), (35) follows from the fact that, if $h(\omega) \in F$, then it belongs to $Y$ and $\|h(\omega)\|_{Y}=\|h(\omega)\|_{F}$ and from the identity

$$
\mathcal{L}^{0}(\Omega, \mathcal{G}, P ; F)=\left\{h: \Omega \rightarrow Y, h \in \mathcal{L}^{0}(\Omega, \mathcal{G}, P ; Y)\right\} .
$$

This identity is a consequence of the two following properties:

- First, if $h$ is measurable into $Y$, it is measurable into $F$ since $\mathcal{B}(F) \subset \mathcal{B}(Y)$ (this imbedding holds because the $\sigma$-algebra $\mathcal{B}$ is generated by closed sets, and every closed set of $F$ is closed in $Y$ ).

- Second, if $h$ is measurable into $F$, it is measurable into $Y$ since, given $B \in \mathcal{B}(Y)$, then $h^{-1}(B)=h^{-1}(B \cap F)$ and $B \cap F \in \mathcal{B}(F)$ (this holds because, if $B$ is closed in $Y$, then $B \cap F$ is closed in $F$ ).

Let us now prove (36) in three steps.

- First,

$$
\mathcal{D}^{\prime}(0, T ; F)=\left\{h \in \mathcal{D}^{\prime}(0, T ; Y): h(\varphi) \in F, \forall \varphi \in \mathcal{D}(0, T)\right\} .
$$

This is obvious since $\mathcal{D}^{\prime}(0, T ; Y)=\mathcal{L}_{c}(\mathcal{D}(0, T) ; Y)$.

- Second,

$$
L^{r}(0, T ; F)=L^{r}(0, T ; Y) \cap \mathcal{D}^{\prime}(0, T ; F) .
$$

Indeed, given mollifiers $\left(\rho_{n}\right)_{n \in \mathbb{N}}$ and a localizing sequence $\left(\alpha_{n}\right)_{n \in \mathbb{N}}$ (that is $\alpha_{n} \in \mathcal{C}^{\infty}, \alpha_{n}=0$ outside $(1 / n, T-1 / n), \alpha_{n}=1$ in $\left.[2 / n, T-2 / n]\right)$, let $h_{n}=\left(\alpha_{n} h\right) \star \rho_{n}$. Then, $h_{n} \in \mathcal{D}(0, T ; F)$, and therefore $\left\|h_{n}-h_{m}\right\|_{L^{r}(0, T ; F)}=$ $\left\|h_{n}-h_{m}\right\|_{L^{r}(0, T ; Y)}$, and $h_{n} \rightarrow h$ in $L^{r}(0, T ; Y)$. Therefore $\left(h_{n}\right)_{n \in \mathbb{N}}$ is a Cauchy sequence in $L^{r}(0, T ; F)$ which is complete, and then $h \in L^{r}(0, T ; F)$. The converse being obvious, (38) holds.

In fact, this proof does not hold if $r=\infty$; in this case, it suffices to remark that $L^{\infty}(0, T ; Y)=\left\{f \in L^{1}(0, T ; Y):\|f\|_{Y} \in L^{\infty}(0, T)\right\}$.

- Third,

$$
W^{s, r}(0, T ; F)=W^{s, r}(0, T ; Y) \cap \mathcal{D}^{\prime}(0, T ; F) .
$$

For $s=0$, it is (38).

For $s>0$, it follows from (38) since $W^{s, r}=\left\{h: \partial_{t}^{n} h \in L^{r}, n \leq s\right\}$. 
For $s=-1$, let $h \in W^{-1, r}(0, T ; Y) \cap \mathcal{D}^{\prime}(0, T ; F)$. By definition, $h=$ $h_{0}+\partial_{t} h_{1}$ where $h_{i} \in L^{r}(0, T ; Y)$, and then $h=\partial_{t} g$ where $g=h_{1}+\int_{0}^{\cdot} h_{0} \in$ $L^{r}(0, T ; Y)$. Let $\xi \in \mathcal{D}(0, T)$ be such that $\int_{0}^{T} \xi=1$, and let $k=g-\int_{0}^{T} g \xi$. Obviously, $k \in L^{r}(0, T ; Y)$. Assume for a moment that

$$
k \in \mathcal{D}^{\prime}(0, T ; F) .
$$

Then, $k \in L^{r}(0, T ; F)$ by $(38)$ and, since $\partial_{t} k=\partial_{t} g=h$, it follows that $k \in W^{-1, r}(0, T ; Y)$. The converse being obvious, (39) holds for $s=-1$.

Now, let us check (40). Given $\varphi \in \mathcal{D}(0, T)$,

$$
\begin{aligned}
\langle k, \varphi\rangle_{\mathcal{D}^{\prime} \times \mathcal{D}} & =\int_{0}^{T}\left(g(t)-\int_{0}^{T} g(s) \xi(s) d s\right) \varphi(t) d t \\
& =\int_{0}^{T} g(t)\left(\varphi(t)-\xi(t) \int_{0}^{T} \varphi(s) d s\right) d t \\
& =\int_{0}^{T} g(t) \partial_{t} \psi(t) d t
\end{aligned}
$$

where $\psi=\int_{0}^{\cdot}\left(\varphi-\xi \int_{0}^{T} \varphi\right)$. But $\psi$ lies in $\mathcal{D}(0, T)$ since it is differentiable, it cancels at 0 and $T$, and it is constant on a neighbourhood of these two points. Then,

$$
\langle k, \varphi\rangle_{\mathcal{D}^{\prime} \times \mathcal{D}}=\left\langle g, \partial_{t} \psi\right\rangle_{\mathcal{D}^{\prime} \times \mathcal{D}}=-\left\langle\partial_{t} g, \psi\right\rangle_{\mathcal{D}^{\prime} \times \mathcal{D}}=-\langle h, \psi\rangle_{\mathcal{D}^{\prime} \times \mathcal{D}}
$$

which lies in $F$ since $h \in \mathcal{D}^{\prime}(0, T ; F)$. With (37), this proves (40).

Finally, a similar proof may be given for $s \leq-2$. It is left to the reader since this case is not used in the present work.

Remark 4.3 In view of the proof of Theorem 4.1, $\left(H^{-1}(D)\right)^{d}$ may be replaced by any Sobolev space $\left(W^{s_{2}, r_{2}}(D)\right)^{d}$ or by $\left(\mathcal{D}^{\prime}(D)\right)^{d}$ in $(26)$, provided that $L^{2}(D)$ be replaced by $W^{s_{2}+1, r_{2}}(D)$ or by $\mathcal{D}^{\prime}(D)$ in $(28)$ and that $\int_{D}$ be replaced by any linear form on this space in (30).

Moreover, Theorem 4.1 extends to any open subset $D$ of $\mathbb{R}^{d}$, instead of assumptions (4), provided that $L^{2}(D)$ be replaced by $L_{\mathrm{loc}}^{2}(D)$ in $(28)$ and that (30) be replaced, for any connected component $D_{i}$ of $D$, by $\int_{d_{i}} p d x=0$, where $d_{i}$ is a non-empty open bounded set in $\mathbb{R}^{d}$ such that $\overline{d_{i}} \subset D_{i}$.

Finally, Theorem 4.1 extends to all $s_{1} \in \mathbb{R}$ by interpolation.

\section{$5 \quad$ Proof of Theorem 2.2.}

Let us remind, see for example [6], that there exists a process $u$ satisfying (11) and (17) - and therefore

$$
u \in L^{2}\left(\Omega, \mathcal{F}_{t}, P ; L^{2}(0, t ; V) \cap L^{\infty}(0, t ; H)\right), \quad \forall t \in(0, T],
$$


- (13) and, $P$-a.s., the variational equation (2) for all $t \in[0, T]$ and $v \in \mathcal{V}$. Differentiating (2) with respect to $t$ ( $\omega \in \Omega$ being fixed), we get, in $\mathcal{D}^{\prime}(0, T)$,

$$
\begin{array}{r}
-\int_{D} \partial_{t} u \cdot v d x-\nu \int_{D} \nabla u \cdot \nabla v d x-\int_{D}((u \cdot \nabla) u) \cdot v d x \\
+\langle F(\cdot, u), v\rangle_{\left.\left.\left(H^{-1}(D)\right)^{d}\right) \times\left(H_{0}^{1}(D)\right)^{d}\right)}+\int_{D} G(\cdot, u) \dot{W}_{t} \cdot v d x=0 .
\end{array}
$$

Since $v \in(\mathcal{D}(D))^{d}$, thanks to (19), (21) and $\langle\nabla u, \nabla v\rangle=-\langle\Delta u, v\rangle$, this reads

$$
\left\langle-\partial_{t} u+\nu \Delta u-(u \cdot \nabla) u+F(\cdot, u)+G(\cdot, u) \dot{W}_{t}, v\right\rangle_{\left(\mathcal{D}^{\prime}(D)\right)^{d} \times(\mathcal{D}(D))^{d}}=0 .
$$

Let us denote $h=-\partial_{t} u+\nu \Delta u-(u \cdot \nabla) u+F(\cdot, u)+G(\cdot, u) \dot{W}_{t}$. As we will check next, (41) implies

$$
h \in L^{1}\left(\Omega, \mathcal{F}_{t}, P ; W^{-1, \infty}\left(0, t ;\left(H^{-1}(D)\right)^{d}\right)\right), \quad \forall t \in(0, T],
$$

therefore Theorem 4.1 provides $p$ satisfying (12), (16) and $\nabla p=h$, that is the Navier-Stokes equation (14).

By (41), $P$-a.s., $u \in L^{2}(0, T ; V)$. Since $\nabla \cdot v=0$ for all $v \in \mathcal{V}$ and therefore, by continuity, for all $v \in V$, this implies $\nabla \cdot u=0$, that is (15).

It only remains to check (43). Denoting $\mathcal{E}$ the space in its right-hand side, let us check that all the terms of $h$ belong to it.

- First, $\partial_{t}$ is linear continuous from $L^{\infty}(0, T ; H)$ into $W^{-1, \infty}(0, T ; H)$ and then into $W^{-1, \infty}\left(0, T ;\left(H^{-1}(D)\right)^{d}\right)$, and therefore $(41)$ implies $\partial_{t} u \in \mathcal{E}$.

- Next, $\Delta$ being linear continuous from $\left(H^{1}(D)\right)^{d}$, and then from $V$, into $\left(H^{-1}(D)\right)^{d},(41)$ implies $\nu \Delta u \in L^{2}\left(\Omega, \mathcal{F}_{T}, P ; L^{2}\left(0, T ;\left(H^{-1}(D)\right)^{d}\right)\right)$ which is included in $\mathcal{E}$ due to the topological imbedding

$$
L^{1}(0, T ; Y) \subset W^{-1, \infty}(0, T ; Y) .
$$

To get this imbedding it suffices to notice that every $f \in L^{1}(0, T ; Y)$ satisfies $f=\partial_{t} \int_{0}^{\cdot} f$ and $\int_{0}^{*} f \in L^{\infty}(0, T ; Y)$, and therefore $f \in W^{-1, \infty}(0, T ; Y)$ thanks to its definition in Section 3.6.

- As seen in Section 3.9, the map $v \mapsto(v \cdot \nabla) v$ is bilinear continuous from $V \times V$ into $\left(L^{4 / 3}(D)\right)^{d}$, and then into $\left(H^{-1}(D)\right)^{d}$ by Sobolev theorem since $d \leq 4$. Therefore, $(41)$ implies $(u \cdot \nabla) u \in L^{1}\left(\Omega, \mathcal{F}_{T}, P ; L^{1}\left(0, T ;\left(H^{-1}(D)\right)^{d}\right)\right)$ which is included in $\mathcal{E}$ by (44).

- Next, $F(\cdot, u) \in \mathcal{E}$ thanks to (7), (8), (9) and (11).

- Finally, $G(\cdot, u) \dot{W}_{t} \in \mathcal{E}$ by (25). This ends the proof of (43). 
Remark 5.1 This proof shows that there exists a positive number $c_{3}(D, T)$ such that any pair $(u, p)$ satisfying (11) to (14) satisfies, in addition, P-a.s.,

$$
\begin{aligned}
& \|p\|_{W^{-1, \infty}\left(0, T ; L^{2}(D)\right)} \leq c_{3}(D, T)\left(\|u\|_{L^{\infty}\left(0, T ;\left(L^{2}(D)\right)^{d}\right)}\right. \\
& \left.\quad+\left(\nu+c_{1}\right)\|u\|_{L^{2}\left(0, T ;\left(H^{1}(D)\right)^{d}\right)}+\left(\|u\|_{L^{2}\left(0, T ;\left(H^{1}(D)\right)^{d}\right)}\right)^{2}\right) \\
& \leq c_{3}(D, T)\left(\|u\|_{L^{\infty}\left(0, T ;\left(L^{2}(D)\right)^{d}\right)}+\left(1+\left(\nu+c_{1}\right)\|u\|_{L^{2}\left(0, T ;\left(H^{1}(D)\right)^{d}\right)}\right)^{2}\right) .
\end{aligned}
$$

It follows that

$$
\begin{array}{r}
\|p\|_{L^{1}\left(\Omega, \mathcal{F}, P ; W^{-1, \infty}\left(0, T ; L^{2}(D)\right)\right)} \leq c_{3}(D, T)\left(\|u\|_{L^{1}\left(\Omega, \mathcal{F}, P ; L^{\infty}\left(0, T ;\left(L^{2}(D)\right)^{d}\right)\right)}\right. \\
\left.+\left(1+\left(\nu+c_{1}\right)\|u\|_{L^{2}\left(\Omega, \mathcal{F}, P ; L^{2}\left(0, T ;\left(H^{1}(D)\right)^{d}\right)\right)}\right)^{2}\right) .
\end{array}
$$

\section{Nonexistence result for a body force valued in $V^{\prime}$.}

From now, the assumptions on the body force $F$ in (7), (8) and (9) are replaced by

$$
\left\{\begin{array}{l}
F \text { is measurable from }[0, T] \times\left(L^{2}(D)\right)^{d} \text { into } V^{\prime} \\
v \mapsto\langle F(t, v), \phi\rangle_{V^{\prime} \times V} \text { is continuous from }\left(L^{2}(D)\right)^{d} \text { into } \mathbb{R}, \\
\|F(t, v)\|_{V^{\prime}} \leq c_{1}\left(1+\|v\|_{\left(L^{2}(D)\right)^{d}}\right) .
\end{array}\right.
$$

Then, with all others assumptions (4) to (10), the proof of the existence of a variational solution given in [6] provides a solution $u$ of (3) instead of (2). It again satisfies (41) and then $f=F(\cdot, u)$ satisfies

$$
f \in L^{2}\left(\Omega, \mathcal{F}, P ; L^{2}\left(0, T ; V^{\prime}\right)\right) .
$$

Let $g=-\partial_{t} u+\nu \Delta u-(u \cdot \nabla) u+G(\cdot, u) \dot{W}_{t}$. Proceeding as in the proof of Theorem 2.2, we get

$$
g \in L^{1}\left(\Omega, \mathcal{F}, P ; W^{-1, \infty}\left(0, T ;\left(H^{-1}(D)\right)^{d}\right)\right)
$$

and, instead of (42),

$$
\langle g, v\rangle_{\left(\mathcal{D}^{\prime}(D)\right)^{d} \times(\mathcal{D}(D))^{d}}+\langle f, v\rangle_{V^{\prime} \times V}=0, \quad \forall v \in V .
$$

The existence of a corresponding pressure is ruled out by the following result. 
Theorem 6.1 There is no Hausdorff locally convex topological vector space in which, for arbitrary $f$ and $g$ satisfying (46), (47) and (48), the equation

$$
g+f=\nabla p
$$

might hold.

Remark 6.2 With the above definitions of $f$ and $g$, (49) reads

$$
-\partial_{t} u+\nu \Delta u-(u \cdot \nabla) u+G(\cdot, u) \dot{W}_{t}+F(\cdot, u)=\nabla p,
$$

that is Navier-Stokes equation (1). Since it cannot hold, (3) should not be named "variational Navier-Stokes equation"; this name should be reserved to (2), the equivalence with (1) being lost as $\left(H^{-1}(D)\right)^{d}$ is replaced by $V^{\prime}$.

Remark 6.3 Equation (48) equivalently reads, thanks to (21),

$$
\langle g, v\rangle_{\left(H^{-1}(D)\right)^{d} \times\left(H_{0}^{1}(D)\right)^{d}}+\langle f, v\rangle_{V^{\prime} \times V}=0 .
$$

Remark 6.4 The reader may be surprised since, in contradiction with Theorem 6.1, various papers contain proofs of the existence of pressure for $V^{\prime}$ valued body forces. These proofs are wrong, the mistake generally lying in the use of a de Rham type theorem to equation (48), as if it was

$$
\langle g+f, v\rangle_{\left(\mathcal{D}^{\prime}(D)\right)^{d} \times(\mathcal{D}(D))^{d}}=0
$$

or $\langle g+f, v\rangle_{\left(H^{-1}(D)\right)^{d} \times\left(H_{0}^{1}(D)\right)^{d}}=0$.

The confusion follows from the use, in these proofs, of the same notation $\langle\cdot, \cdot\rangle$ for the duality products in $\left(\mathcal{D}^{\prime}(D)\right)^{d} \times(\mathcal{D}(D))^{d}$, in $\left(H^{-1}(D)\right)^{d} \times$ $\left(H_{0}^{1}(D)\right)^{d}$ and in $V^{\prime} \times V$.

Proof of Theorem 6.1. Would such a space, say $X$, exist, it should contain $L^{2}\left(\Omega, \mathcal{F}, P ; L^{2}\left(0, T ; V^{\prime}\right)\right)$ and $L^{1}\left(\Omega, \mathcal{F}, P ; W^{-1, \infty}\left(0, T ;\left(H^{-1}(D)\right)^{d}\right)\right)$, at least in the sense that there should be two linear injective maps $i$ and $j$ from these spaces into $X$. Then a linear injective map $\widehat{i}$ from $V^{\prime}$ into $X$ would be defined by $\widehat{i} \varphi=i \widehat{\varphi}$ where $(\widehat{\varphi}(\omega))(t)=\varphi$ for all $\omega$ and $t$. Similarly, we would define a linear injective map $\widehat{j}$ from $\left(H^{-1}(D)\right)^{d}$ into $X$.

Since each $\psi \in\left(H^{-1}(D)\right)^{d}$ defines a unique $Q \psi \in V^{\prime}$ by

$$
\langle Q \psi, v\rangle_{V^{\prime} \times V}=\langle\psi, v\rangle_{\left(H^{-1}(D)\right)^{d} \times\left(H_{0}^{1}(D)\right)^{d}}, \quad \forall v \in V,
$$

then $\psi$ and $Q \psi$ should correspond to the same element of $X$, that is

$$
\widehat{j} \psi=\widehat{i} Q \psi .
$$


Consider now $\phi=\nabla q$, for a given non constant $q \in L^{2}(D)$. Then, $\phi \in$ $\left(H^{-1}(D)\right)^{d}, \quad \phi \neq 0$, and $Q \phi=0$ since $\langle\phi, v\rangle_{\left(H^{-1}(D)\right)^{d} \times\left(H_{0}^{1}(D)\right)^{d}}=$ $-\langle q, \nabla \cdot v\rangle_{\left(\mathcal{D}^{\prime}(D)\right)^{d} \times(\mathcal{D}(D))^{d}}=0$ for all $v \in \mathcal{V}$ and thus, by continuity, for all $v \in V$. Then, $\widehat{i} Q \phi=0$ and therefore $\widehat{j} \phi=0$ would hold, in contradiction with the injectivity of $\hat{j}$.

The map $Q$ defined by (50) is "canonical" since $Q \psi$ is the restriction to $V$ of the map $\psi \in \mathcal{L}_{c}\left(\left(H_{0}^{1}(D)\right)^{d} ; \mathbb{R}\right)$. Let us summarize its properties.

Lemma 6.5 The map $Q$ is linear continuous from $\left(H^{-1}(D)\right)^{d}$ onto $V^{\prime}$ and is not one to one.

Proof. Continuity holds since, by (50) and (20), $\|Q \psi\|_{V^{\prime}} \leq\|\psi\|_{\left(\left(H_{0}^{1}(D)\right)^{d}\right)^{\prime}} \leq$ $\|\psi\|_{\left(H^{-1}(D)\right)^{d}}$ (thanks to the definition of $H^{-1}$, this holds for $c=1$ ).

The range of $Q$ is $V^{\prime}$ since by Hann-Banach theorem, any $\varphi \in V^{\prime}$ possesses an extension $\psi \in\left(\left(H_{0}^{1}(D)\right)^{d}\right)^{\prime}$, and then $Q \psi=\varphi$.

It is not one to one since, in the proof of Theorem 6.1 , we built $\phi \neq 0$ such that $Q \phi=0$.

Let us now give strong equations that, instead of Navier-Stokes one, are satisfied by solutions of (3). First, in $V^{\prime}$, we have, $P$-a.s.,

$$
Q\left(\partial_{t} u-\nu \Delta u+(u \cdot \nabla) u\right)=F(\cdot, u)+Q\left(G(\cdot, u) \dot{W}_{t}\right)
$$

in $\mathcal{D}^{\prime}\left(0, T ; V^{\prime}\right)$. This is not totally satisfactory since pressure disappeared.

The other possibility is to give an equation in $\left(H^{-1}(D)\right)^{d}$. There exist infinitely many $\Phi \in L^{1}\left(\Omega, \mathcal{F}, P ; L^{1}\left(0, T ;\left(H^{-1}(D)\right)^{d}\right)\right)$ such that $Q \Phi=F(\cdot, u)$ (for example, a solution is $\Phi=\Delta S(F(\cdot, u))$ where $S f$ is, for a given $f \in V^{\prime}$, the solution of $\int_{D} \nabla S f \cdot \nabla v=\langle f, v\rangle_{V^{\prime} \times V}$ for all $\left.v \in V\right)$. For such a $\Phi$, there exists $p \in L^{1}\left(\Omega, \mathcal{F}, P ; W^{-1, \infty}\left(0, T ;\left(H^{-1}(D)\right)^{d}\right)\right)$ such that, $P$-a.s.,

$$
\partial_{t} u-\nu \Delta u+(u \cdot \nabla) u+\nabla p=\Phi+Q\left(G(\cdot, u) \dot{W}_{t}\right)
$$

in $\mathcal{D}^{\prime}\left(0, T ;\left(H^{-1}(D)\right)^{d}\right)$. This again is not satisfactory, now since $\Phi$ and $p$ are not unique (even if $p$ is normalized by $\int_{D} p=0$ ); worst, all $p$ in the above space can be associated to a given $u$. Indeed, given a pair $\left(\Phi_{0}, p_{0}\right)$ satisfying (52), the pair $\left(\Phi_{0}+\nabla\left(p-p_{0}\right), p\right)$ is another solution since $Q\left(\Phi_{0}+\nabla\left(p-p_{0}\right)\right)=$ $Q \Phi_{0}=F(\cdot, u)$ because, as seen in the proof of Theorem $6.1, Q \nabla q=0$ for all $q$. 
Remark 6.6 The set spanned by $u$ is the same, wether forces span $V^{\prime}$ or $\left(H^{-1}(D)\right)^{d}$, since $Q$ defined by (50) maps $\left(H^{-1}(D)\right)^{d}$ onto $V^{\prime}$. (Surjectivity follows from $V \subset\left(H_{0}^{1}(D)\right)^{d}$ since their topologies coincide on $V$; a strict imbedding $V^{\prime} \subset W^{\prime}$ holds when $W$ is a dense subset of $V$ equipped with a strictly finer topology, that is not the case here).

The only effect of choosing forces in $V^{\prime}$ instead of $\left(H^{-1}(D)\right)^{d}$ is to suppress information on pression, since $Q^{-1}$ may be viewed as a one to one map from $V^{\prime}$ onto $\left(H^{-1}(D)\right)^{d} / \nabla L^{2}(D)$. Indeed, given $f \in V^{\prime}$ and $g_{0} \in Q^{-1} f$,

$$
\begin{gathered}
Q^{-1} f=\left\{g \in\left(H^{-1}(D)\right)^{d}:\left\langle g-g_{0}, v\right\rangle_{\left(H^{-1}(D)\right)^{d} \times\left(H_{0}^{1}(D)\right)^{d}} \text { for all } v \in V\right\} \\
=\left\{g_{0}+\nabla q: q \in L^{2}(D)\right\} .
\end{gathered}
$$

This explains why Navier-Stokes equation, which contains information on pressure, cannot contain terms lying in $V^{\prime}$.

Acknowledgements. The authors acknowledge E. Fernández Cara for pointing out the necessity of clarifying the existence of the pressure in the solution of the stochastic Navier-Stokes equations.

\section{References}

[1] Bensoussan A (1995) - Stochastic Navier-Stokes equations, Acta Appl. Math. 38, 3: 267-304

[2] Bensoussan A, Temam R (1973) - Equations stochastiques du type Navier-Stokes, J. Funct. Anal. 13: 195-222

[3] Brzeźniak Z, Capiński M, Flandoli F (1992) - Stochastic Navier-Stokes equations with multiplicative noise, Stochastic Anal. Appl. 10, 5: 523532

[4] Capiński M, Cutland N (1991) - Stochastic Navier-Stokes equations, Acta Appl. Math. 25: 59-85

[5] Capiński M, Cutland N (1993) - Navier-Stokes equations with multiplicative noise, Nonlinearity 6: 71-77

[6] Capiński M, Peszat S (2001) - On the existence of solution to stochastic Navier-Stokes equations, Nonlinear Anal. Theory Methods Appl. 44: $141-177$ 
[7] DaPrato G, Zabczyk J (1992) - Stochastic equations in infinite dimensions, Cambridge University Press, Cambridge

[8] Lions JL (1965) — Problèmes aux limites dans les équations aux dérivées partielles, Presses de l'Université de Montreal

[9] Schwartz L (1973) - Théorie des distributions, Hermann, 1961, new edition, Paris.

[10] Simon J (1993) - Démonstration constructive d'un théorème de G. de Rham, C. R. Acad. Sci. Paris, sér. I 316: 1167-1172

[11] Simon J (1993) - Representation of distributions and explicit antiderivatives up to the boundary, in Progress in partial differential equations: the Metz surveys 2, M. Chipot ed., Longman, 201-205

[12] Simon J (1999) - On the existence of the pressure for solutions of the variational Navier-Stokes equations, J. Math. Fluid Mech. 1: 225-234 